\title{
Pharmacological treatment of neuropathic pain in older persons
}

\author{
Clair Haslam' \\ Turo Nurmikko² \\ 'The Walton Centre for Neurology \\ and Neurosurgery, Liverpool, England, \\ UK; ${ }^{2}$ The Pain Research Institute, \\ Division of Neurological Science, \\ University of Liverpool, Liverpool, \\ England, UK
}

\begin{abstract}
Interest and research into the mechanisms and treatment of neuropathic pain have increased during recent years, but current treatment is still far from satisfactory (Dworkin et al 2003; Attal et al 2006). The European Federation of Neurological Societies (EFNS) Task Force recently published guidelines for the pharmacological treatment of neuropathic pain (Attal et al 2006). However, no particular consideration is given as to how the recommendations are applicable to the elderly population. This paper will review the guidelines in relation to this population and evaluate the existing evidence relating to the use of these drugs in older persons.
\end{abstract}

Keywords: neuropathic pain, elderly, anticonvulsants, antidepressants, opioids, tramadol, lidocaine patch/plaster, capsaicin

\section{Background}

There is currently a major shift occurring in the age distribution of the world's population. In developed countries, the proportion of the population aged over 65 will rise from $17.5 \%$ to $36.3 \%$ by 2050 and the over- 80 age segment will more than triple (US Bureau of the Consensus, International Data, 2002). There is no clear consensus as to the definition of an "older person". However, it is suggested that further categorization of those over 65 may be useful: the young old can be defined as 65-74 years, the middle old as 75-84 years and the oldest old as 85 and older (Leppik 2006). However, there can be vast differences in people of the same chronological age. Therefore, there are also suggestions that each age group should be further sub-divided into: healthy, multiple medical problems and frail (Leppik 2006).

Pain is known to be a common problem in the elderly population, but it frequently goes unrecognized and under-treated (Helme and Gibson 1999; IASP 2006). The situation is complicated further by the increased potential for cognitive impairment and communication problems in the elderly (IASP 2006). Such is the concern about this issue that the International Association for the Study of Pain (IASP) made 2006/07 the IASP "Global Year Against Pain in Older Persons" in an attempt to raise awareness and improve treatment. This subject is also part of their Core Curriculum for pain education. It has been identified that there is an age-related increase in the prevalence of chronic pain at least until the seventh decade of life (Helme and Gibson 2001). Studies suggest that chronic pain affects over $50 \%$ of older persons living in the community setting and over $80 \%$ of nursing home residents (Ferrell 1995; Helme and Gibson 2001).

Neuropathic pain results from disease or injury affecting the peripheral nerve, nerve root, spinal cord or brain. A large number of pathophysiological mechanisms have been delineated, and there is general agreement that in many persistent cases several of them are operant in maintaining the pain. Pain is commonly described as burning, shooting, smarting or aching, and local tenderness to mechanical and cool stimuli is common. The hallmark signs of neuropathic pain are reduced sensibility
Correspondence: Clair Haslam The Walton Centre, Lower Lane, Fazakerley, Liverpool, England L9 7LJ, UK Tel +44 I5I 5295647

Email clair.haslam@thewaltoncentre.nhs.uk 
and increased sensitivity corresponding to the site of pain (Treede et al 2007). The newly formulated definition of neuropathic pain, "pain arising as a direct consequence of a lesion or disease affecting the somatosensory system" encompasses the important pathophysiological aspect that because of impairment of sensory pathways at some level the clinical examination is likely to show sensory abnormalities (Treede et al 2007). The diagnosis of neuropathic pain is based on evidence of a lesion present corresponding to the site of pain, and irrespective of commencement of treatment, etiological investigations are sometimes required.

Specific epidemiological data on neuropathic pain is limited. However, there is an estimated prevalence of $1.5 \%$ (Taylor 2006). In addition, there is a wide range of prevalence estimates reported in the literature for specific conditions. Between $8 \%$ and $50 \%$ of diabetics are estimated to have symptoms of diabetic neuropathy (Duck et al 1993; Partanen et al 1995). In a community survey of over 300 patients with diabetes the prevalence of painful neuropathy was $16 \%$ (Daousi et al 2004). Between $10 \%$ and 19\% of back pain sufferers are estimated to have neuropathic pain (Carey et al 1996; Loney and Stratford 1999). In a study of 916 patients with shingles, $18 \%$ of patients in their 50 s experienced symptoms of post-herpetic neuralgia for 1 year or longer. However, in patients aged 70 or above this increased to $48 \%$ (Oaklander and Rissmiller 2002).

\section{Treatment of neuropathic pain in the elderly}

The treatment of neuropathic pain in the older person can be challenging and complex. The old are more vulnerable than younger persons since they often suffer from multiple medical and nutritional problems. This can limit treatment options with analgesic agents due to an increased risk of adverse effects and problems with complex drug interactions (Perucca et al 2006). In addition, older persons may have substantial differences in the absorption and clearance of drugs. Due to these factors there is an increased risk of excess sedation, confusion, constipation and urinary retention (Burris 2004).

A detailed literature review of pharmacokinetics in older persons found that changes occur due to age-related physiologic perturbations (Cusack 2004). However, the review suggests that age-related changes may be overshadowed by individual variation in disease, frailty and stress. It is also acknowledged that older persons take more medications and are more likely to have multiple medical problems, and this increases the risk of drug-drug and drug-disease interactions (Cusack 2004). It is suggested that the rate of drug metabolism, which occurs mainly in the liver, is often reduced with advancing age. In drugs that are mainly excreted by the kidney the rate of elimination is also generally reduced in the elderly. Therefore, the dose requirement for these types of drugs may be lower in many older patients (Cusack 2004).

Despite the fact the chronic pain is more common in older persons, the majority of pain treatment studies and trials have been conducted in young adult populations (IASP 2006). Until recently older subjects were routinely excluded from randomized controlled trials of pharmacotherapy. Even now, older people with co-morbid disease or those taking other medication are usually excluded (IASP 2006). Therefore, it is possible to suggest that there is significant selection bias and that the "healthy" elderly subjects who are included in studies are not representative of the general elderly population. In particular it is acknowledged that most gerontologic studies of pharmacokinetics fail to include the very old (age 85 and over) and therefore the understanding of pharmacokinetics in this age group is very limited (Cusack 2004).

Several recent systematic reviews provide convergent guidance on pharmacological management of neuropathic pain (Dworkin, Backonja et al 2003; Finnerup et al 2005; Attal et al 2006; Moulin et al 2007). The recommendations based on these reviews come from randomized controlled trials from which it is usually difficult to extract information specific to elderly patients. The general recommendation is to commence treatment with gabapentin/pregabalin or a tricyclic antidepressant (eg, amitriptyline or nortriptyline) and, in case of focal neuropathy, topical lidocaine. The choice between a gabapentinoid and a tricyclic is based on considerations on tolerability rather than quality or intensity of pain. Second line treatments include SNRIs (duloxetine and venlafaxine), some antiepileptic drugs (eg, lamotrigine), tramadol and topical capsaicin. Reservations are expressed in relation to strong opioids which feature as second or third line choices. Despite the general agreement between the three Task Force generated guidelines (Dworkin, Backonja et al 2003; Attal et al 2006; Moulin et al 2007), there are some differences as shown in Table 1.

The EFNS Guidelines (Attal et al 2006) discuss treatment options in specific neuropathic conditions, ie, painful polyneuropathies, post-herpetic neuralgia (PHN), trigeminal neuralgia (TN) and central pain (including spinal cord injury pain, post-stroke pain and multiple sclerosis). In addition, 
Table I Commonly used agents in neuropathic pain: choice of treatment as recommended by three task forces

\begin{tabular}{|c|c|c|c|c|}
\hline Drug & Dworkin et al 2003 & Attal et al 2006 & Moulin et al 2007 & Comment \\
\hline TCA & 1 & 1 & I & \\
\hline Gabapentin & 1 & 1 & 1 & \\
\hline Pregabalin & 1 & 1 & 1 & \\
\hline Tramadol & I & 2 & 3 & \\
\hline Duloxetine & NR & 2 & 2 & \\
\hline Venlafaxine & 2 & 2 & 2 & \\
\hline Lamotrigine & 2 & 2 & 4 & \\
\hline Opioids & 1 & 2 & $3^{\prime}$ & 'Except methadone \\
\hline $\begin{array}{l}\text { Lidocaine 5\% } \\
\text { plaster }\end{array}$ & 1 & $2^{2}$ & 2 & $\begin{array}{l}{ }^{2} \text { First-line in focal } \\
\text { neuropathy with } \\
\text { allodynia }\end{array}$ \\
\hline Topical capsaicin & 3 & $2^{3}$ & 3 & ${ }^{3} \mathrm{PHN}$ only \\
\hline Valproate & NR & 2 & 4 & \\
\hline Topiramate & NR & $\mathrm{NE}$ & 4 & \\
\hline Bupropion & 2 & NR & NR & \\
\hline Paroxetine & 2 & $\mathrm{NR}^{4}$ & NR & $\begin{array}{l}{ }^{4}+\text { ve RCT } \\
\text { acknowledged }\end{array}$ \\
\hline Citalopram & 2 & NE & 4 & \\
\hline Mexiletine & 3 & $\mathrm{NE}$ & 4 & \\
\hline Clonidine & 3 & $\mathrm{NE}$ & 4 & \\
\hline $\begin{array}{l}\text { NMDA } \\
\text { antagonists }\end{array}$ & 3 & $\mathrm{NE}$ & NR & \\
\hline Methadone & as other opioids & As other opioids & 4 & \\
\hline \multicolumn{5}{|l|}{ Specific indications } \\
\hline Carbamazepine for TGN & 1 & I & 1 & $\begin{array}{l}\text { No guideline } \\
\text { recommends for } \\
\text { other NPs }\end{array}$ \\
\hline Oxcarbazepine for TGN & NR & I & NR & \\
\hline $\begin{array}{l}\text { Cannabinoids for central } \\
\text { pain in MS }\end{array}$ & NR & 3 & 4 & \\
\hline
\end{tabular}

Note: 'first-line treatment, ${ }^{2}$ second-line treatment, ${ }^{3}$ third-line treatment, ${ }^{4}$ fourth-line treatment. Note that only Moulin et al (2007) use the category of "fourth-line". $\mathrm{NR}$, no recommendation or no discussion; NE, considered not effective.

other less studied neuropathic conditions are included: post-traumatic/post-surgical nerve lesions, phantom limb pain and Guillain-Barré syndrome. In general, because of the few condition-specific controlled trials published, the authors suggest management along the same lines as those used in diabetic neuropathy and post-herpetic neuralgia for which ample data are available. Notable exceptions are lamotrigine in post-stroke pain, pregabalin in spinal cord injury pain, carbamazepine and oxcarbazepine in trigeminal neuralgia and cannabinoids in central pain related to multiple sclerosis (Attal et al 2006). In none of the comprehensive reviews are there age-specific recommendations, although reference to poorer tolerability in the elderly is occasionally made. The authors have generated their own approach to pharmacotherapy for the elderly with neuropathic pain (Table 2). It is a compilation of systematic reviews and guidelines with some modifications that reflect safety concerns in this patient population.
Currently, the best evidence available suggests that the choice of medication in the elderly with neuropathic pain should be similar to that in the younger age group but commenced more cautiously and with particular attention to problems that may arise in the elderly. In this review we will discuss each drug in relation to the information and evidence available for its use in the elderly. We have drawn from two sources: from studies focusing on the elderly with other indications (eg, depression, epilepsy) or extrapolating from studies on postherpetic neuralgia in which the mean age of the participating patients has been $>70$ years.

\section{Antidepressants}

A recent review of the literature on the extent to which the elderly are represented in clinical trials of potential antidepressants found that elderly subjects aged 75 years and over were clearly under-represented (Giron et al 2005). The maximum age for inclusion for the majority of the clinical 
Table 2 Drug treatment of older persons with neuropathic pain

\section{First line treatment}

Tricyclic antidepressants (amitriptyline, nortriptyline)

Gabapentinoids (gabapentin, pregabalin)

Lidocaine plaster $5 \%$

Carbamazepine and oxcarbazepine in $\mathrm{TN}^{2}$

Second line treatment

Tramadol

Lamotrigine

SNRIs (venlafaxine, duloxetine)

\section{Third line treatment}

Opioids (morphine, oxycodone, methadone) ${ }^{3}$

Cannabinoids in $\mathrm{MS}^{4}$

Citalopram and paroxetine $e^{5}$

Capsaicin

Note: 'In patients with no or stable cardiac conditions only

${ }^{2}$ Trigeminal neuralgia

${ }^{3}$ Despite efficacy, concerns regarding tolerability make these drugs third line only

${ }^{4}$ Data on elderly patients extremely limited

${ }^{5}$ In peripheral neuropathies only

trials identified was 65 years. There was no clear consensus on the definition of elderly; clinical trials that were reported to have been conducted in the elderly included subjects who were 50,55 , or 60 years and over. The highest age reported for a subject was 90 years. Pharmacological studies on healthy subjects were mainly done on young adults ranging from 18 to 65 years. Therefore, there is minimal evidence available in the literature about the safety and efficacy of antidepressants in older persons, whether they are being used to treat depression and/or pain. The authors of the review (Giron et al 2005) recommend that regulatory bodies such as the Food and Drug Administration (FDA) and European Agency for the Evaluation of Medicinal Products (EMEA) should have special requirements relating to the elderly. For example, the inclusion of at least $25 \%$ of subjects aged 75 years and over in studies of drugs that will be registered for use in the elderly.

Due to the fact that the majority of studies of antidepressants use a highly selective trial population, without significant co-morbidity and with minimum numbers of elderly subjects, there are fundamental problems extrapolating the results to the elderly population as a whole (Parikh 2000). However, despite the lack of evidence, there are some recommendations in the literature relating to the use of antidepressants in the elderly. It is widely reported that older patients are more prone to side-effects and have greater difficulty tolerating doses of antidepressants that are likely to be of therapeutic value (Parikh 2000; ANZCA 2005). Older adults often require lower initial doses with slow titration and the initial dose should be one-half or lower than the normal starting dose (Antai-Otong 2006).

\section{Tricyclic antidepressants (TCAs)}

A recent study found a slight increase in sudden cardiac death with TCA doses greater than $100 \mathrm{mg}$ per day (Ray et al 2004). The study examined a retrospective cohort of 1487 patients with confirmed sudden cardiac death in the community. Current users of TCAs had a dose-related increase in the risk of sudden cardiac death when compared with non-users of antidepressants. Rate ratios increased from 0.97 (95\% confidence interval (CI), 0.72-1.29) for doses lower than $100 \mathrm{mg}$ (amitriptyline or equivalent) to 2.53 (95\% CI, 1.04-6.12) for doses of $300 \mathrm{mg}$ or more. The rate for SSRIs was 0.95 (95\% CI, 0.42-2.15). There was no evidence that TCA doses lower than $100 \mathrm{mg}$ increased the risk of sudden cardiac death in subgroups defined by pre-existing cardiovascular disease, female sex, age 65 or older or use of amitriptyline. Although from this study the conclusion is that low doses of amitriptyline (or equivalent) may be used with a maximum dose of $100 \mathrm{mg}$, other well known anticholinergic, hypotensive and sedative side effects may pose a relative contraindication to tricyclic antidepressants, and a safer alternative in the very old may be gabapentin or pregabalin (Attal et al 2006). Due to the risk of potential life-threatening side-effects some authors recommend baseline and follow-up electrocardiograms (ECGs) throughout treatment with tricyclic antidepressants (Dworkin, Backonja et al 2003). In Parikh's (2000) advice on the posology of antidepressants in the elderly a lower starting and maintenance dose is advised for amitriptyline, but no specific recommendations are made for nortriptyline.

In a head-to-head cross-over comparison study of tricyclic antidepressants and strong opioids for the treatment of PHN, 71 evaluable patients (mean age (SD) 71 (12) y) were allowed to self-titrate to maximum tolerated dose of 160mg nortriptyline per day. A mean (SD) dose 89 (27) mg per day was better tolerated than morphine 91 (49) $\mathrm{mg}$ per day (Raja et al 2002). Two patients withdrew during nortriptyline treatment as opposed to seven during morphine treatment, and constipation, nausea and drowsiness were less common. However, 17\% reported dizziness, significantly more than when receiving morphine. Nortriptyline, however, showed a trend toward lesser efficacy compared with morphine.

Due to the potential toxicity and adverse effects of TCAs, especially in the elderly population, consideration of dosing 
levels is important. Dose-effect and serum concentration-effect relationships have been reported in the literature. However, it is suggested that serum concentration-effect relationships may be more appropriate due to the large inter-individual variations in pharmacokinetics (Sindrup 2003). In a randomized, controlled trial of amitriptyline and carbamazepine in central post-stroke pain it was found that total plasma levels of amitriptyline varied greatly (56-925 nmol/1) despite the fact that all patients had tolerated the planned final dose of $75 \mathrm{mg} /$ day (Leijon and Boivie 1989). The pain-relieving effect of amitriptyline appeared to correlate well with the total plasma concentration. Of patients with plasma concentration exceeding $300 \mathrm{nmol} / 1,70 \%$ were responders on the daily rating of the effect and $90 \%$ were responders on the global rating. This is compared to only $20 \%$ responders at plasma concentrations below $300 \mathrm{nmol} / \mathrm{l}$. This would indicate that a total plasma concentration of $300 \mathrm{nmol} / 1$ should be used as a cut off point for the lowest effective drug level of amitriptyline when it is being used to treat neuropathic pain. This is lower than the reported corresponding level for the treatment of depression which is $500 \mathrm{nmol} / \mathrm{l}$ (Sindrup 2003).

\section{Serotonin-noradrenaline reuptake inhibitors (SNRIs)}

There is general agreement that SNRIs such as duloxetine and venlafaxine are safer to use than TCAs and are a better option in patients with cardiac disease. However, the risk of hyponatraemia due to the syndrome of inappropriate secretion of antidiuretic hormone (SIADH) is thought to be greater in elderly patients using selective serotonin re-uptake inhibitors (SSRIs)/SNRIs than those using TCAs (Antai-Otong 2006). Therefore, it is recommended that baseline electrolyte levels are checked and that sodium levels are closely monitored throughout treatment. The Committee on Safety of Medicines (CSM) has advised that hyponatremia should be considered in all patients who develop drowsiness, confusion or convulsions while taking an antidepressant (BNF 2007).

In a recent observational, naturalistic, multicenter, prospective, open-label study of 97 patients older than 80 years with depressive syndrome, extended-release venlafaxine was found to be safe and effective (Baca et al 2006). At baseline, $90.7 \%$ of the patients had additional medical conditions and $83.5 \%$ were receiving concomitant medications. Adverse events were reported by $7(7.2 \%)$ patients, but no serious adverse events were reported. The most frequent adverse events were dizziness, gastric pain and nausea. Treatment with venlafaxine over 24 weeks did not produce any clinically significant changes in blood pressure, heart rate or other variables such as weight. Baca et al (2006) suggest that venlafaxine is particularly useful in the treatment of the elderly due to a low potential for drug-drug interaction. This study provides valuable information because the study sample more accurately reflects the typical elderly population.

\section{Antiepileptic drugs}

Antiepileptic drugs (AEDs) form an important part of the pharmacological management of neuropathic pain, but as is the case with TCAs they require a good deal of vigilance on the part of the prescribing clinician. Sodium channels blockers, especially carbamazepine and oxcarbazepine are effective in trigeminal neuralgia, but have well known major side effects. Their usefulness is limited in other neuropathic conditions (Attal et al 2006). A particular area of concern in the elderly is the risk of hyponatraemia with carbamazepine and oxcarbazepine. It is recommended that sodium levels should be monitored in the first few months of treatment and that both drugs should be initiated with low dosages and slowly increased.

Further information on the use of AEDs in the elderly comes from studies on the treatment of epilepsy. AEDs are one of the main classes of drugs most commonly implicated as causing adverse drug reactions in the elderly (Perucca et al 2006). Available information on the pharmacokinetic properties of AEDs refers mainly to patients aged 70 or less, despite the fact that there is a high prevalence of AED use among elderly patients, for both the treatment of epilepsy and other indications such as neuropathic pain (Perucca et al 2006).

A recent paper reviewed two completed studies of AED use in the elderly and one still in progress (Leppik et al 2006). The first was a multicenter, randomized, doubleblind comparison between carbamazepine and lamotrigine in elderly patients with newly diagnosed epilepsy (Brodie at al 1999). No difference was noted between the drugs in time to first seizure. However, in relation to the use of these drugs in neuropathic pain it is the additional data on adverse events that is of interest. Fewer patients taking lamotrigine (18\%) dropped out of the study due to adverse events, compared to the patients taking carbamazepine (42\%). Somnolence was reported significantly more often in patients taking carbamazepine (29\%) than those taking lamotrigine $(12 \%, \mathrm{p}<0.01)$.

The other completed study was a three-arm, doubleblind, randomized clinical trial in patients aged 65 years or older with new-onset seizures, comparing the newer AEDs, gabapentin and lamotrigine, to old standard AED therapy 
with carbamazepine (Rowan et al 2005). 593 patients met the inclusion criteria, but 570 of those were male because the sample was taken from the United States Veterans Affairs (VA) system. This means that there is a significant male gender bias in the study sample and therefore the results may not be generalizable to the elderly female population. Differences in early discontinuation among the drugs were apparent by 7 weeks. The most frequent discontinuations were with carbamazepine $(27.3 \%)$, followed by gabapentin( $17.4 \%$, $\mathrm{p}<0.01)$ and then by lamotrigine $(10 \%, \mathrm{p}<0.00003)$. The primary outcome measure was retention in the study at 52 weeks. Two hundred and seventy six patients $(46.5 \%)$ completed the trial. Of those patients who withdrew from the study, $131(41.3 \%)$ discontinued treatment due to adverse reactions to the study drug, and $33(10 \%)$ due to uncontrolled seizures. These results concur with the findings of a previous multi-centre double-blind trial in 150 elderly patients with newly diagnosed epilepsy, which found that lamotrigine was better tolerated than carbamazepine (Brodie et al 1999). These results provide some justification for the choice of gabapentin and lamotrigine in elderly patients in certain neuropathic pain conditions in which efficacy is shown. However, in case of trigeminal neuralgia, which commonly affects people over 65 , the superior efficacy of carbamazepine over gabapentin and lamotrigine dictates its choice as first line treatment.

Leppik (2005) suggests that for an AED to be ideal for the elderly it should have the following characteristics: no side effects or toxicity, complete oral absorption (related to water solubility), no drug-drug interactions, long half-life, no hepatic metabolism or enzyme induction/inhibition, no active metabolites, minimal protein binding, no effects on bone loss and a reasonable price. In general, the early AEDs available before 1980 have significant potential for side-effects because they deviate from the ideal (Leppik 2005). Gastrointestinal drug absorption is altered with aging and AEDs that are highly insoluble (eg, phenytoin, carbamazepine) are generally poorly absorbed and complicate dosing regimes (Leppik 2005). Lamotrigine is more soluble than the early AEDs and gabapentin is water soluble, but it relies on a rate-limiting transport system for absorption and has dose-dependent oral absorption (Leppik 2005). However, it is possible to suggest that both these drugs are closer to the ideal than the early AEDs.

Recommendations have been made in relation to the selection of AEDs for older patients with epilepsy and these can be applied to the treatment of older patients with neuropathic pain. Gabapentin and pregabalin are identified as two of the newer AEDs that have the fewest drug interactions. These drugs exert no significant effects on other medications likely to be used by the elderly and are not affected by other medications in terms of their serum levels or rate of metabolism (Leppik 2005). This is important because many elderly patients are on multiple medications and this increases the risk of drug-drug interactions. However, lamotrigine is metabolized by the liver and may have reduced levels when given with enzyme inducers (Leppik 2005).

Many newer AEDs also have better safety profiles than the older agents and gabapentin, pregabalin and lamotrigine have not had associated liver or bone marrow toxicity (Leppik 2005). Oxcarbazepine is considered to be safer than carbamazepine in relation to the fact it has no epoxide metabolite, has less hepatic induction and it does not depress white cell counts. The aplastic anemia and agranulocytosis that can be seen with carbamazepine may also not occur (Leppik 2005). However, the incidence of hyponatremia is higher with oxcarbazepine than carbamazepine (Bergey 2004; Leppik 2005). It is suggested that oxcarbazepine should be avoided in elderly patients taking sodiumdepleting agents (eg, diuretics) (Bergey 2004). Gabapentin and pregabalin are both renally excreted and dose reductions should be made if there is significant renal insufficiency (Bergey 2004).

Cognitive side-effects are also of particular concern in the elderly because they may be more sensitive to small changes due to pre-existing cognitive dysfunction, multiple medications and concomitant medical problems (Leppik 2005). Gabapentin, pregabalin and lamotrigine are classed as some of the newer AEDs that appear to have particularly favorable cognitive profiles compared with the older drugs (Leppik 2005). However, even these drugs do cause significant central nervous system side effects, especially dizziness and drowsiness, which not infrequently lead to discontinuation of treatment. In older individuals the firstline AED should be one that causes less cognitive sideeffects, because these effects may often go unrecognized, particularly in patients with communication problems, or they may be attributed to other problems such as Alzheimer's or cerebrovascular disease (Bergey 2004). Both gabapentin and pregabalin have been studied in PHN (Rowbotham et al 1998; Rice et al 2001; Dworkin, Corbin et al 2003; Sabatowski et al 2004). The mean age in these studies varied between 71 and 76 years, with the oldest participants in their 90s. In one study $81 \%$ of the patients were over 65 . The highest doses for gabapentin were $3600 \mathrm{mg} /$ day and for pregabalin $600 \mathrm{mg} /$ day. In the study by Rowbotham et al (1998), 83\% 
tolerated a dose of at least $2400 \mathrm{mg} / \mathrm{day}$, and $13.3 \%$ in the gabapentin group withdrew from the study versus $9.5 \%$ in the placebo group. Pregabalin at doses of $300 \mathrm{mg} /$ day and higher led to withdrawal of $32 \%$ due to adverse effects (as opposed to $5 \%$ in the placebo group) but no drug-related serious adverse effects were reported (Dworkin, Corbin, et al 2003). It is unclear from these reports if the frequency and intensity of side effects correlated with age. Both gabapentin and pregabalin are effective in reducing pain in PHN with numbers-needed-to-treat (50\%) estimated at $4.6(95 \%$ CI 3.7-6.0) (Finnerup et al 2005).

\section{Tramadol}

Tramadol hydrochloride is a synthetic analgesic that has a dual mechanism of action. It has a central analgesic effect with a weak affinity for the $\mu$-opioid receptor and also causes spinal inhibition of the reuptake of noradrenaline and serotonin (Budd 1999; Schnitzer 1999). The EFNS Guidelines (Attal et al 2006) recommend that tramadol should be initiated at low dosages of $50 \mathrm{mg}$ once daily in elderly patients and then titrated as tolerated. A dose adjustment is not usually necessary in elderly patients (up to 75 years) without clinically manifest hepatic or renal insufficiency (Schnitzer 1999). In elderly patients (over 75 years) elimination may be prolonged. Therefore, if necessary, the dosage interval is to be extended according to the patients requirements. Tramadol can induce convulsions and increase the potential for SSRIs, TCAs, anti-psychotics and other seizure threshold-lowering medicinal products to cause convulsions. In isolated cases there have been reports of serotonin syndrome in a temporal connection with the therapeutic use of tramadol in combination with other serotonergic medicinal products such as selective serotonin SSRIs or with MAO inhibitors (Grunenthal 2006). It is essential that these precautions are taken into consideration when tramadol is being used in elderly patients because they are often taking multiple medications which increase the risk of drug-drug interactions and adverse effects.

A randomized, double-blind, placebo-controlled trial was recently carried out to evaluate the efficacy and safety of a combined paracetamol $325 \mathrm{mg}$ and tramadol $37.5 \mathrm{mg}$ tablet for the treatment of flare-ups of osteoarthritis pain in elderly patients (aged 65 and older) (Rosenthal et al 2004). The patients were permitted to take one to two tablets four times daily. The combination was found to be significantly superior to placebo for average daily pain intensity and pain relief. The most common treatment related adverse events in the tramadol/paracetamol group were nausea (18.8\%), vomiting (13\%), dizziness (11.6\%) and constipation (4.3\%). The low incidence of constipation ( $4.3 \%$ vs $2.3 \%$ in placebo group) and somnolence ( $2.9 \%$ vs $2.3 \%$ in placebo group) were worthy of note. The study concludes that the tolerability profile of this combination medication, especially the low rates of constipation and somnolence, make it an attractive alternative to traditional opioid analgesics for elderly patients. However, the duration of the study was only 10 days and therefore one must exercise caution in extrapolating these results to long-term use in chronic pain conditions.

\section{Strong opioids}

Strong opioids are increasingly being used for patients with chronic benign pain. In 2005 the British Pain Society published recommendations for the appropriate use of opioids for persistent non-cancer pain, but no specific guidance is offered in relation to elderly patients. Strong opioids have also been shown to be effective in the treatment of chronic neuropathic pain (Gimbel et al 2003; Watson et al 2003). The EFNS guidelines (Attal et al 2006) recommend their use as second-line treatment in a number of neuropathic pain conditions, but again no specific guidance is provided for their use in elderly patients. It is suggested that elderly patients may require less opioid than younger patients and in the post-operative setting there is evidence of an age-related 2-4 fold decrease in morphine and fentanyl requirements (Macintyre and Jarvis 1996; Woodhouse and Mather 1997; Gagliese et al 2000).

Elderly patients who are commenced on strong opioids require regular monitoring because reduced renal function can lead to a more rapid accumulation of active opioid metabolites (ANZCA 2005). Both morphine and oxycodone produce active metabolites that can accumulate causing increased therapeutic and adverse effects (Johnson 2007). It is recommended that both drugs are used cautiously in patients with renal dysfunction, with careful monitoring and dose adjustment if necessary. Fentanyl has no active metabolites, but a dose reduction is still advised because clearance is reduced in patients with moderate to severe uremia (Johnson 2007).

There is a paucity of research in the literature on the use of strong opioids in elderly patients with chronic pain. However, one recent longitudinal study of 10,372 nursing home residents concluded that the use of long-acting opioids may be a relatively safe option in the management of persistent, non-malignant pain, yielding benefits in functional status and social engagement (Won et al 2006). A significantly higher rate of improvement in activities 
of daily living (ADL) status and social engagement was observed in patients taking long-acting opioids compared to those taking non-opioids. There were no significant declines in cognitive function, ADL status, mood status or social engagement over 6 months with the use of any analgesics. Overall, a trend towards a decreased risk for falls over 6 months was seen with the use of any analgesics. Long-term use of opioids did not significantly increase the risk of falls. The authors acknowledge that the study has limitations because it was necessary to restrict the sample to persons taking analgesics in only one class for 6 months; whereas in reality, many patients may take analgesics from a number of different classes. However, this is the only study identified that examines quality of life outcomes of chronic analgesic use in frail elderly persons.

In the two studies published on the efficacy of morphine and oxycodone, the benefit from moderately high doses (mean dose $89 \mathrm{mg}$ of morphine and $45 \mathrm{mg}$ of oxycodone) of both drugs was well documented with a joint NNT of 2.6 (95\% CI 2.0-3.8) (Finnerup et al 2005). Common side effects included constipation nausea, drowsiness, and loss of appetite but no cognitive impairment was reported (Watson and Babul 1998; Raja et al 2002). Side effects led to withdrawal in over one-quarter of patients (mean age (SD) 71 (12) years) on morphine despite its apparent superiority over placebo and nortriptyline. While tolerability in studies with long up-and-down titration protocols and a short (2week) maintenance period give some insight of the efficacy (Raja et al 2002), it is not well known if effectiveness continues long-term in this condition and to what extent dose adjustment is needed.

\section{Topical agents}

Topical capsaicin is seen as a modestly effective treatment in PHN and diabetic neuropathy (Bernstein et al 1989; Watson et al 1993). To maintain the effect, the cream must be applied over the painful area several times a day and initially causes severe burning, leading to at least $30 \%$ of patients stopping treatment prematurely. It is possible to suggest that the use of capsaicin in elderly frail patients with cognitive impairments may not be advisable due to the potential for inadvertent exposure of other areas of the body. If capsaicin was accidentally rubbed into the eyes or onto other mucous membranes significant irritation and discomfort/distress would be caused.

A topical agent recently licensed in the United Kingdom (UK) for the treatment of PHN is the 5\% lidocaine plaster. Due to its low systemic absorption this treatment is considered safe and only local adverse effects (mild skin reactions) have been reported. Several authors recommend it is used as first-line treatment in patients with PHN with a small area of pain / allodynia and state that it may be preferred as a treatment for elderly patients due to excellent tolerability (Finnerup et al 2005; Attal et al 2006; Moulin et al 2007). A recent review of the literature concludes that the safety, tolerability, and efficacy of the lidocaine patch 5\%, a targeted peripheral analgesic, has been well established in PHN (Gammaitoni et al 2003). The review reports that potential efficacy in other neuropathic and non-neuropathic pain conditions has also been suggested in recent case reports and studies. Several pharmacokinetic studies have demonstrated minimal systemic absorption with 12-, 18-, and 24-hour/day dosing. Due to the low mean maximum plasma concentrations there is minimal risk for systemic toxicity or drug-drug interactions. The most common adverse events generally involve mild skin reactions and there have been no drug-drug interactions noted in clinical trials (Gammaitoni et al 2003). All these factors make this an ideal drug for use in the elderly and it appears that the risk of adverse events is minimal even in those with multiple medical problems and concomitant medication use. However, there is only sufficient evidence in the literature at the current time for the use of the 5\% lidocaine plaster in the treatment of PHN and it is only licensed in the UK for this indication. It is currently recommended for use in PHN and focal neuropathy (Finnerup et al 2005; Attal et al 2006), but not for all neuropathic pain conditions.

\section{Conclusion}

Pharmacotherapy remains the mainstay of management of neuropathic pain in the elderly. There is a relative paucity of studies available from which specific guidelines can be produced. However, indirect evidence suggests that current guidelines aimed at the neuropathic pain population in general can also be adopted for the older person's treatment, given that potential alterations in pharmacokinetics and pharmacodynamics of various drugs are taken into account and contraindications and tolerability issues are strictly observed. In general, drugs should be commenced at a lower dose and be upwardly titrated more slowly than in middle-aged and young people. Studies in PHN, which include a majority of patients who are elderly, show clinically significant efficacy from tricyclic antidepressants, opioids and gabapentinoids and moderate-to-good tolerability in most cases. Reports of serious side effects typical for the elderly are very few. Tricyclic antidepressants warrant a word of caution because of their cardiotoxicity and other problems. In general, studies in the treatment of neuropathic pain in the elderly, other than PHN, and especially studies in 
the oldest-old that evaluate the effectiveness and tolerability of current and new drugs are desperately needed.

\section{References}

Antai-Otong D. 2006. Antidepressants in late-life depression: prescribing principles. Perspectives in Psychiatric Care, 42:149-53.

Attal N, Cruccu G, Haanpaa M, et al. 2006. EFNS guidelines on pharmacological treatment of neuropathic pain. European Journal of Neurology, 13:1153-69.

[ANZCA] Australian and New Zealand College of Anaesthetists. 2005. Acute pain management: scientific evidence. 2nd ed. Australia: National Health and Medical Research Council.

Baca E, Roca M, Garcia-Calvo C, et al. 2006. Venlafaxine extended-release in patients older than 80 years with depressive syndrome. International Journal of Geriatric Psychiatry, 21:337-43.

Bergey G. 2004. Initial treatment of epilepsy: special issues in treating the elderly. Neurology, 63:S40-8.

Bernstein J, Korman N, Bickers D, et al. 1989. Topical capsaicin treatment of chronic postherpetic neuralgia. Journal of American Academy of Dermatology, 21:265-70.

Brodie M, Overstall P, Giorgi L. 1999. Multicentre, double-blind, randomised comparison between lamotrigine and carbamazepine in elderly patients with newly diagnosed epilepsy. The UK Lamotrigine Elderly Study Group. Epilepsy Research, 37:81-7.

British National Formulary. 2007. BNF 53.

Budd K. 1999. The role of tramadol in acute pain management. Acute Pain, 2:189-96.

Burris J. 2004. Pharmacologic approaches to geriatric pain management Archives of Physical Medicine and Rehabilitation, 85(Suppl 3):S45-9.

Carey T, Evans A, Hadler N, et al. 1996. Acute severe low back pain. A population-based study of prevalence and care-seeking. Spine, 21:339-44.

Cusack B. 2004. Pharmacokinetics in Older Persons. The American Journal of Geriatric Pharmacotherapy, 2:274-302.

Daousi C, MacFarlane IA, Woodward A, et al. 2004. Chronic painful peripheral neuropathy in an urban community: a controlled comparison of people with and without diabetes. Diab Med, 21:976-82.

Duck P, Krtaz K, Karnes L, et al. 1993. The prevalence by staged severity of various types of diabetic neuropathy, retinopathy, and neuropathy in a population-based cohort: The Rochester Diabetic Neuropathy Study. Neurology, 43:817-24.

Dworkin R, Backonja M, Rowbotham M. 2003. Advances in neuropathic pain: diagnosis, mechanisms and treatment recommendations. Archives of Neurology, 60:1524-34.

Dworkin RH, Corbin AE, Young JP, et al. 2003. Pregabalin for the treatment of postherpetic neuralgia. A randomized, placebo-controlled trial Neurology, 60:1274-83.

Ferrell B. 1995. Pain evaluation and management in the nursing home Annals of Internal Medicine, 123:681-7.

Finnerup NB, Otto M, McQuay HJ, et al. 2005. Algorithm for neuropathic pain treatment: an evidence based approach. Pain, 118:289-305.

Gagliese L, Jackson M, Ritvo P, et al. 2000. Age is not an impediment to effective use of patient-controlled analgesia by surgical patients. Anesthesiology, 93:601-10.

Gammaitoni A, Alvarez N, Galer B. 2003. Safety and tolerability of the lidocaine patch 5\%, a targeted peripheral analgesic: a review of the literature. Journal of Clinical Pharmacology, 43:111-17.

Gimbel J, Richards P, Portenoy R. 2003. Controlled release oxycodone for pain in diabetic neuropathy. A randomised controlled trial. Neurology, 60:927-34

Giron M, Fastbom J, Winblad B. 2005. Clinical trials of potential antidepressants: to what extent are the elderly represented: a review. International Journal of Geriatric Psychiatry, 20:201-17.

Helme R, Gibson S. 1999. Chapter 8: pain in older people. In: Crombie I ed. Epidemiology of pain. Seattle: IASP Press. p 103-12.
Helme R, Gibson S. 2001. The epidemiology of pain in elderly people. Clinical Geriatric Medicine, 17:417-31.

International Association for the Study of Pain. 2006. Older people's pain. Pain Clinical Updates, 14:1-4.

Johnson S. 2007. Opioid safety in patients with renal or hepatic dysfunction Pain treatment topics [online]. Accessed 1 June 2007. URL: http://www. pain-topics.org.

Leijon G, Boivie J. 1989. Central post-stroke pain - a controlled trial of amitriptyline and carbamazepine. Pain, 36:27-36.

Leppik I. 2005. Choosing an antiepileptic: selecting drugs for older patients with epilepsy. Geriatrics, 60(11):42-7.

Leppik I. 2006. Antiepileptic drug trials in the elderly. Epilepsy Research, 68:45-8.

Leppik I, Brodie M, Saetre E. 2006. Outcomes research: clinical trials in the elderly. Epilepsy Research, 68S:S71-6.

Loney P, Stratford P. 1999. The prevalence of low back pain in adults: a methodological review of the literature. Physical Therapy, 79:384-96.

Macintyre P, Jarvis D. 1996. Age is the best predictor of postoperative morphine requirement. Pain, 64:357-64.

Moulin DE, Clark AJ, Gilron I, et al. 2007. Pharmacological management of chronic neuropathic pain - consensus statement and guidelines from the Canadian Pain Society. Pain Res Manage, 12:13-21.

Oaklander A, Rissmiller J. 2002. Post-herpetic neuralgia after shingles is an under-recognised cause of chronic vulvar pain. Obstetrics and Gynaecology, 99:625.

Parikh C. 2000. Antidepressants in the elderly: challenges for study design and their interpretation. British Journal of Clinical Pharmacology, 49:539-47.

Partanen J, Niakanen L, Lehiten J, et al. 1995. Natural history of peripheral neuropathy in patients with non-insulin dependent diabetes mellitus. New England Journal of Medicine, 333:88-94.

Perucca E, Berlowitz D, Birnbaum A, et al. 2006. Pharmacological and clinical aspects of antiepileptic drug use in the elderly. Epilepsy Research, 68S:S49-63.

Raja SN, Haythornethwaite, Pappagallo M, et al. 2002. Opioid versus antidepressants in postherpetic neuralgia. A randomized controlled trial. Neurology, 59:1015-21.

Ray W, Meredith S, Thapa P, et al. 2004. Cyclic antidepressants and the risk of sudden cardiac death. Clinical Pharmacology and Therapeutics, $75: 234-41$.

Rice ASC, Maton S. 2001. Postherpetic Neuralgia Study Group. Gabapentin in postherpetic neuralgia: a randomised, double blind, placebo controlled study. Pain, 94:215-24.

Rosenthal N, Silverfield J, Wu S, et al. 2004. Tramadol/acetaminophen combination tablets for the treatment of pain associated with osteoarthritis flare in an elderly patient population. Journal of the American Geriatric Society, 52:374-80.

Rowan A, Ramsay R, Collins J, et al. 2005. VA Cooperative Study 428 Group. New onset geriatric epilepsy: a randomised study of gabapentin, lamotrigine, and carbamazepine. Neurology, 64:1868-73.

Rowbotham M, Harden N, Stacey B, et al. 1998. Gabapentin for the treatment of postherpetic neuralgia. JAMA, 280:1837-42.

Sabatowski R, Galvez R, Cherry DA, et al. 2004. Pregabalin reduces pain and improves sleep and mood disturbances in patients with post-herpetic neuralgia: results of a randomised, placebo-controlled clinical trial. Pain, 109:26-35.

Schnitzer T. 1999. Managing chronic pain with tramadol in elderly patients. Clinical Geriatrics, 7(8).

Sindrup S. 2003. Chapter 18: Antidepressants and chronic pain. In: Jensen T, Wilson P, Rice A eds. Clinical pain management: chronic pain. London: Arnold.

Taylor R. 2006. Epidemiology of refractory neuropathic pain. Pain Practice, 6:22-6.

Treede R-D, Jensen TS, Campbell JN, et al. In press. Neuropathic pain: redefinition and a grading system for clinical and research diagnostic purposes. Neurology. 
US Bureau of the Consensus 2002. International Database.

Watson C, Tyler K, Bickers D, et al. 1993. A randomised vehicle-controlled trial of topical capsaicin in the treatment of postherpetic neuralgia. Clinical Therapeutics, 15:510-26.

Watson C, Babul N. 1998. Efficacy of oxycodone in neuropathic pain. A randomized trial in postherpetic neuralgia. Neurology, 50:1837-41.

Watson C, Moulin D, Watt-Watson J, et al. 2003. Controlled-release oxycodone relieves neuropathic pain: a randomised controlled trial in painful diabetic neuropathy. Pain, 105:71-8.
Won A, Lapane K, Vallow S, et al. 2006. Long-term effects of analgesics in a population of elderly nursing home residents with persistent nonmalignant pain. The Journals of Gerontology, 61A:165-9.

Woodhouse A, Mather L. 1997. The influence of age upon opioid use in the patient-controlled analgesia (PCA) environment. Anaesthesia, 52:949-55. 